\title{
Image Fusion Algorithm based on Sparse Features and Pulse Coupled Neural Networks in Wavelet Transform Domain
}

\author{
TIANYunXiang ${ }^{1, a}$, TIANXiaoLin $^{2, b}$ \\ ${ }^{1}$ Facultyof Information Technology,Macau University of Science and Technology, China \\ ${ }^{2}$ Lunar and Planetary Science Laboratory/Space Science Institute, Macau University of Science and \\ Technology, China

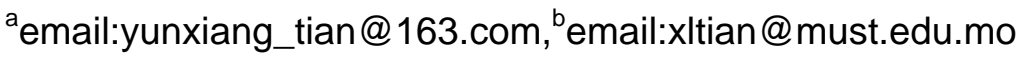

Keywords:Image fusion,robust principal component analysis (RPCA), pulse coupled neural networks (PCNN), wavelet transform

\begin{abstract}
Multi-focus image fusion has become an advanced research topic in image processing field. The traditionalmethods that are commonly used wavelet transform, principal component analysis (PCA),pulse coupled neural networks (PCNN), which got some results, but they are not effective enough for focused regions. In this paper, an algorithm based on sparse features and pulse coupled neural networks in wavelet transform (WT) domain has been proposed. Robust principal component analysis (RPCA) combined PCNN are applied to low frequency of the wavelet coefficients, and spatial frequency (SF) combined PCNN are applied to high frequency components. The experiment results show that the method proposed not only improve the quality of the fused image, but also preserved clarity and information, which gives better results than the other techniques used.
\end{abstract}

\section{Introduction}

Multi-focus image fusion is the process of combining two or more images of a scene to create a single image that is more informative than any of the input images ${ }^{[1]}$. Due to the fact that commonly used optical lenses suffer from a problem of limited depth of field, images being captured are not in focus everywhere ${ }^{[2]}$. The out of focus regions are blur. It necessary to process the multi-focus images into an informative image. Multi-focus image fusion has been widely used in various fields, such as computer vision, remote sensing, digital imaging and microscopic imaging ${ }^{[2]}$.

The multi-focus image fusion methods are commonly classified into spatial domain fusion algorithms and frequency domain fusion algorithms. The spatial domain fusion methods operate the image pixels or regions directly, which are easy to calculate. Li et al. ${ }^{[3]}$ used spatial frequency (SF) as the block fusion rules and got good result. Tao et al. ${ }^{[2]}$ decomposed the source images into low rank matrix and sparse matrix by robust principal component analysis (RPCA) method and calculated the SF in block in sparse matrix and reconstructed the image by blocks. But spatial domain fusion methods would cause obviously blocking artifacts and sliding window cost higher computation.Transform domain fusion algorithms convert the source image into frequency domain. Then use different fusion rules to deal with subband coefficients. Finally, reconstruct the fused image via an inverse transformation. There are many advantages in transform domain fusion algorithms, such as gaining more detailed information, avoiding blocking artifacts, increasing fusion quality. Qu et al. ${ }^{[4]}$ proposed a SF motivated pulse coupled neural networks (PCNN) method, which used same rules in all subbands lead to details information loss.

In this paper, a method based on sparse features and PCNN in wavelet transform domain is proposed. Use RPCA method to decompose images high frequency coefficients into low rank matrix and sparse matrix, and calculate SF with each matrix. Then the feature matrixes are used to PCNN neurons. And SF rules are used to low frequency coefficients. The proposed method can give better results and more detailed information in fused image. 


\section{Robust Principal Component Analysis}

In the field of image processing and computer vision, PCA is widely used in high-dimensional data processing, such as data analysis and dimensionality reduction,etc. To deal with the main purpose of the high-dimensional data is effective estimation of high-dimensional data lower dimensional linear space. But when the sparse high-dimensional data contains a lot of noise, it's applicable to the traditional PCA methods no longer. The researchers introduced the RPCA methods to solve the problem.

RPCA is an effective way to recover both low-rank and sparse components exactly form high dimensional data by solving the principal component pursuit ${ }^{[5]}$. When the source data has Gaussian noise, the problem can be solved by singular value decomposition (SVD) through PCA method. But when the sparse high-dimensional data contains spikes noise, it's inapplicable to the traditional PCA methods. So the problem can be solved by RPCA method and described as follow:

$$
M=L_{0}+S_{0}(1)
$$

M: the input data, $\mathrm{L}_{0}$ : low rank matrix, $\mathrm{S}_{0}$ : sparse spikes noise matrix.

It is obvious that the problem is difficult to solve. But Wright et al. ${ }^{[6]}$ proved that sparse matrix $\mathrm{S}_{0}$ is sparse enough relative to the low rank matrix $\mathrm{L}_{0}$, which can be accurately recover low rank matrix $_{0}$ form the source data $\mathrm{M}$ matrix by solving the convex optimization problem,

$$
\min \left\|L_{0}\right\|_{*}+\lambda\left\|S_{0}\right\|_{1}(2)
$$

$$
\text { st. } L_{0}+S_{0}=M
$$

$\|$. $\|_{*}$ denote the low rank matrix $L_{0}$ nuclear norm, $\|.\|_{1}$ represent the sparse matrix $S_{0} L_{1}$ norm, $\lambda$ is a positive weighting parameter.

When $\lambda=1 / \sqrt{\max (m, n)}$ the low rank matrix recovery best. Through the superiority of the RPCA method, we try to acquire the image information more representative in frequency domain,which we can improve the quality of fused image.

\section{Pulse Coupled Neural Network}

PCNN is a feedback network and each PCNN neuron consists of three parts: the receptive field, the modulation field, and the pulse generator ${ }^{[7]}$. In image processing, PCNN is a single layer pulse coupled neural cells with a two-dimensional connection ${ }^{[8]}$. The PCNN method can get some good results in image fusion. If we put the image gray value into the PCNN directly, it will be not effective enough. So in this paper, we put the source image into frequency domain firstly, thenuse RPCA method to deal with high frequency coefficients into low rank matrix and sparse matrix and calculate the SF with each matrix.Finallyput the feature matrix intothe PCNN networks.

The SF is defined in wavelet domain as:

$$
S_{i j}^{k}=\sum_{i \in M, j \in N}\left[\left(I_{i j}^{k}-I_{i-1, j}^{k}\right)^{2}+\left(I_{i j}^{k}-I_{i, j-1}^{k}\right)^{2}\right](3)
$$

The neuron can be described as ${ }^{[4]}$ :

$$
\begin{aligned}
& F_{i j}^{k}(n)=S_{i j}^{k} \\
& F_{i j}^{k}(n)=\exp \left(-\alpha_{L}\right) L_{i j}^{k}(n-1)+V_{L} \sum_{p q} W_{i j, p q} Y_{p q}^{k}(n-1) \\
& U_{i j}^{k}(n)=F_{i j}^{k}(n) *\left(1+\beta L_{i j}^{k}(n)\right)(4) \\
& \theta_{i j}^{k}(n)=\exp \left(-\alpha_{\theta}\right) \theta_{i j}^{k}(n-1)+V_{\theta} Y_{i j}^{k}(n-1)
\end{aligned}
$$


The firing times is calculated as:

$$
Y_{i j}^{k}(n)= \begin{cases}1, & \text { if }: U_{i j}^{k}(n)>\theta_{i j}^{k}(n) \\ 0, & \text { otherwise }\end{cases}
$$

$$
T_{i j}^{k}(n)=T_{i j}^{k}(n-1)+Y_{i j}^{k}(n)(5)
$$

In this PCNN model, $\mathrm{n}$ denotesthe iterations, $I_{i j}^{k}$ denotes the frequency coefficients in K-th subbands in (i,j) position, $U_{i j}^{k}$ is internal activity in neuron, $W_{i j, p q}$ is the synaptic gain strength, $L_{i j}^{k}$ denotes input of connections with neurons, $\theta_{i j}^{k}$ is the threshold and $\beta$ is the linking strength. $\alpha_{L}$ is decay constant. $V_{L}, V_{\theta}$ are scaling terms. If $U_{i j}^{k}$ is larger than $\theta_{i j}^{k}$, the $Y_{i j}^{k}=1$, it means the neuron generates one firing time. The sum of $\mathrm{n}$ iterations is defined as firing times with $T_{i j}^{k}$.

\section{Fusion Algorithm}

In order to deal with the image b] Low Frequency and make up the disadvantages of the space transform, we convertthe source image. ............., domain by WT. Use different fusion rules in high frequency and low frequency coefficients. Instead of using PCNN in WT domain directly, RPCA and spatial frequency is considered as the features of images input into PCNN networks.And we use the firing times to generate the decision map to select WT coefficients.The fusion framework is shown in Fig1,the main stepsof the fusionscheme are:

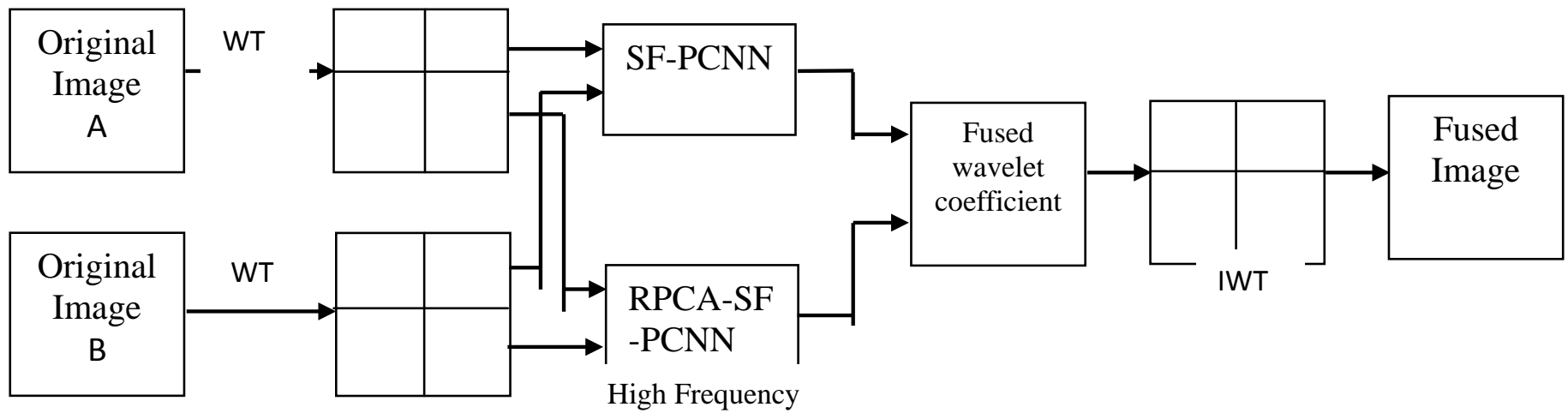

Fig. 1 Flowchart of the proposed fusion algorithm

Step 1: Convertthe source images into frequency domain by WT.

Step 2: Calculate SF inlow frequency coefficients. Then put the feature matrix into the PCNN networks and the firing times $T_{i j}^{k}(n)$ is calculatedin each subband.The decision map can be described as (6).

$$
D_{i j}^{k}(n)=\left\{\begin{array}{l}
1, \text { if }: T_{1, i j}^{k}(n) \geq T_{1, i j}^{k}(n)(6) \\
0, \text { otherwise }
\end{array}\right.
$$

Step 3: Use RPCA method to deal with two images high frequency coefficients into low rank matrixes and sparse matrixes, andcalculate SF with each matrix as the same way in step 2 . Then put the feature matrix into the PCNN networks and the firing times $T_{i j}^{k}(n)$ is calculated.Add new low rank matrixes and sparse matrixes to get newfrequency coefficients in each subband.

Step 4: Construct the new image using newcoefficients are selected by inverse WT.

\section{Experimental Results}

In order to evaluate the performance of the proposed method in this paper, we did some experiments as Fig2 and Fig3.The db4 wavelet basis was selected for the WT process. The parameters of PCNN are $\alpha_{L}=1, \alpha_{\theta}=0.2, V_{L}=1, V_{\theta}=20, \beta=3, n=200$. SF-PCNN ${ }^{[4]}$, wavelet-domain statistics (WS) ${ }^{[9]}$ and laplace method are compared to proposed method. 


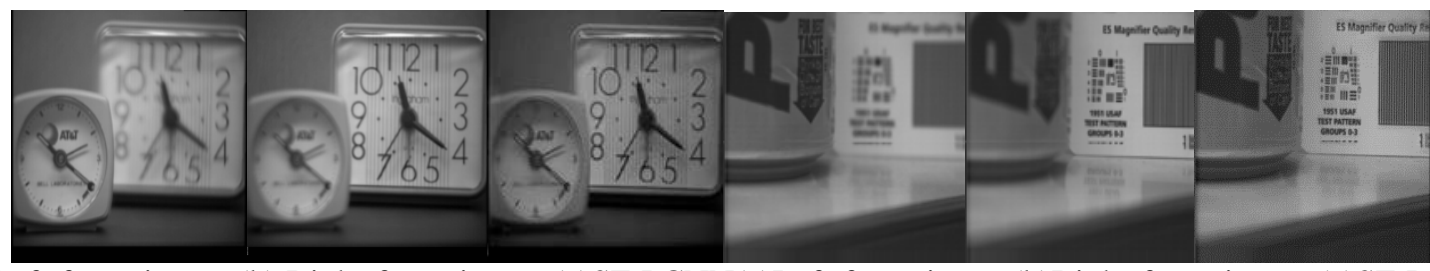

(a) Left-focus image (b) Right-focus image (c)SF-PCNN(a)Left-focus image(b)Right-focus image (c)SF-PCNN

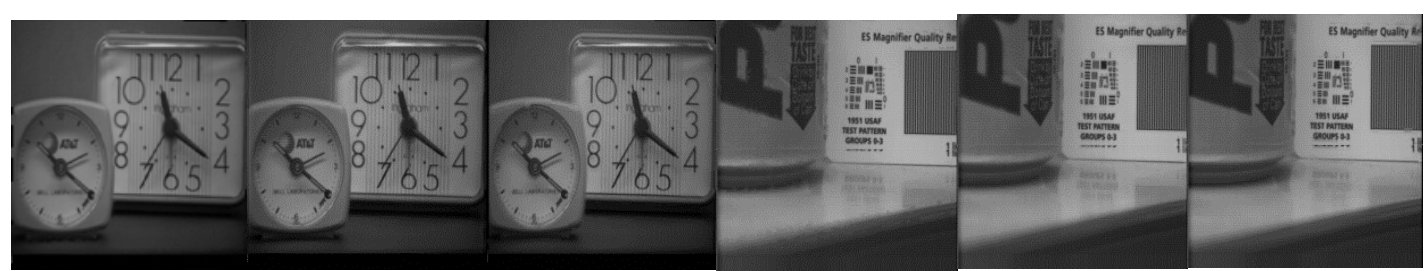

(d)WS

(e)Laplace(f)Proposed method(d)WS (e)Laplace

(f)Proposed method

Fig. 2Image fusion experiment 1Fig. 3Image fusion experiment 2

The experimental results can be seen as shown above.The fused image which used proposedmethod in this paper is more conform to human visual perception. In Fig 2, (f) is clearer than other three fused images on the left edge of the clock.The Fig 2 and Fig 3 (d) which used WS are obvious distortion. The (f) in Fig 3 has more detailed information. The bottle in Fig 3 (e) and (f) is more clearly than the same feature in (c) and (d). The edge and details are blur in Fig 3 (c) which used SF-PCNN method. It is easy to see that the proposed method gives better results and more detailed information than the other techniques used.

The mutual information $(\mathrm{MI})^{[10]}, \mathrm{Q}^{\mathrm{AB} / \mathrm{F}[11]}$ and correlation coefficient three image quality criteria are performed to provide quantitative evaluations that are shown in table 1 . The proposed method obtains largest $\mathrm{MI}$ and relatively largerQ $\mathrm{Q}^{\mathrm{AB} / \mathrm{F}}$ compared to other methods. It means that our method provide more edge and details information of the fused image.However it need higher computational cost.

Table 1. Various performance evaluation of experimental results

\begin{tabular}{|c|c|c|c|c|c|c|c|}
\hline $\begin{array}{c}\text { Fusion } \\
\text { algorithm }\end{array}$ & MI & $\mathrm{Q}^{\mathrm{AB} / \mathrm{F}}$ & $\mathrm{CC}$ & $\begin{array}{c}\text { Fusion } \\
\text { algorithm }\end{array}$ & $\mathrm{MI}$ & $\mathrm{Q}^{\mathrm{AB} / \mathrm{F}}$ & $\mathrm{CC}$ \\
\hline \multicolumn{5}{|c|}{ Experiment 1} & \multicolumn{5}{c|}{ Experiment 2} \\
\hline SF-PCNN & 7.4948 & 0.6775 & 0.9797 & SF-PCNN & 7.8201 & 0.7326 & 0.9828 \\
\hline WS & 8.0441 & 0.6931 & 0.9774 & WS & 7.3136 & 0.7425 & 0.9814 \\
\hline Laplace & 7.1494 & 0.6972 & 0.9792 & Laplace & 7.0256 & 0.7763 & 0.9823 \\
\hline $\begin{array}{c}\text { Proposed } \\
\text { method }\end{array}$ & 8.1229 & 0.6960 & 0.9776 & $\begin{array}{c}\text { Proposed } \\
\text { method }\end{array}$ & 8.0273 & 0.7561 & 0.9815 \\
\hline
\end{tabular}

\section{Conclusion}

This paper proposes a new fusion method based on sparse features and pulse coupled neural networks in wavelet transform domain. The proposed method gives better results than the other techniques used both in quantitative evaluations and qualitative evaluations. The method not only improves the quality of the fused image but alsopreserves more detailed information at the same time. However, the method would cost a lot of computation time and memory. We will reduce the computational in the future.

\section{References}

[1] B. Khaleghi, A, khamis : Multisensor data fusion A review of the state of the art, Information Fusion, Vol. 14 (2013), p. 28-44. 
[2] Tao Wan, Zengchang Qin, Chenchen Zhu, Renjie Liao: A robust fusion scheme for multifocus images using sparse features, Speech and Signal Processing IEEE International Conference(2013), p. 1957-1961.

[3]Shuotao Li, James T, YaonanWang :Combination of images with diverse focuses using the spatial frequency,Information Fusion .(2001),p.169-176.

[4] XiaobuQu, Jingwen Wang, Hongzhi Xiao, ZiqianZhu :Image fusion algorithm based on spatial frequency -motivated pulse coupled neural networks in nonsubsampledcontourlet transform domain, ACTA AutomaticaSinica.(2008),p.1508-1514.

[5] Candes E J, Li X, Ma Y, et al..: Robust principal component analysis?Journal of the ACM,(2011),58:1-37.

[6] Wright J, A Ganesh, S Rao, Ma Y :Robust Principal Component Analysis: Exact Recovery ofCorrupted Low-Rank Matrices via Convex Optimization. Advances in Neural Information Processing Systems.(2009)

[7] J.L. Johnson, M.L. Padgett :PCNN models and applications. IEEE Transactions on Neural Networks, (1999),10(3),p. 480-498.

[8] R.P. Broussard, S.K. Rogers, M.E. Oxley, G.L. Tarr : Physiologically motivated image fusion for object detection using a pulse coupled neural network. IEEE Transaction on Neural Networks, (1999),10(3),p.554-563

[9] JinTian, Li Chen: Multi-focus image fusion using wavelet domain statistics. IEEE International Conference on Image Processing (2010), p. 1205-1208.

[10] D. MacKay, Information Theory, Inference, and Learning Algorithms,Cambridge University Press, (2003).

[11] C. S.Xydeas, V. Petrovic, Objective Image Fusion PerformanceMeasure, Electronics Letters, 36, 308-09, (2000). 